\title{
Dynamic Composite Cladding
}

Dynamic Composite Cladding is a collaborative research project that attempts to recast typical low cost cladding as a high performance, customized system.

JEFFERSON ELLINGER

University of North Carolina, Charlotte

\section{INTRODUCTION}

Dynamic Composite Cladding is a collaborative research project that attempts to recast typical low cost cladding as a high performance, customized system. The focus of this research is to produce a next generation cladding system that departs from the conventional environmental and aesthetic thinking for a building facade. The composite cladding is designed to be a dynamically responsive yet inert facade system with a very low cost of production and installation. While the primary challenge for the system may be understood as being an environmentally responsive wall assembly without mechanical actuation - the aesthetic 'performance' is positioned as an equal priority by applying complex visual design logics as surface topology.

The research emerged out of a partnership established between an advanced composites manufacturing company, an architecture firm and the academy. The tripartite relationship has been critical in the effort to actualize the project and test on two buildings and one interior to date. Students, research/practice faculty and the architecture firm worked with the manufacturer to develop proprietary material compositions, layup schedules and molds for each custom application. The architecture firm worked with the manufacturer and students to understand economies and satisfy client concerns while achieving the diverse and often times competing goals. This progression of research and student collaboration includes several various stages of experimentation ranging from undergraduate course research to graduate research assistantship studies to internships within architecture firms.

\section{BACKGROUND}

Towards the end of the twentieth century computation began to be utilized within the discipline of architecture in a number of ways; however, it was not until late in the century when computation begin to emerge as a tool for analyzing building performance during the design process. Within this trajectory, only recently has the availability of computational power reached a point to which Computational Fluid Dynamic (CFD) simulation could be widely used to analyze performance at building scale. Here the early building CFD studies 


\section{Biological Case Studies surface articulation}


For the Saguaro cactus, the grooves serve functionally for load and heat transfer reduction.

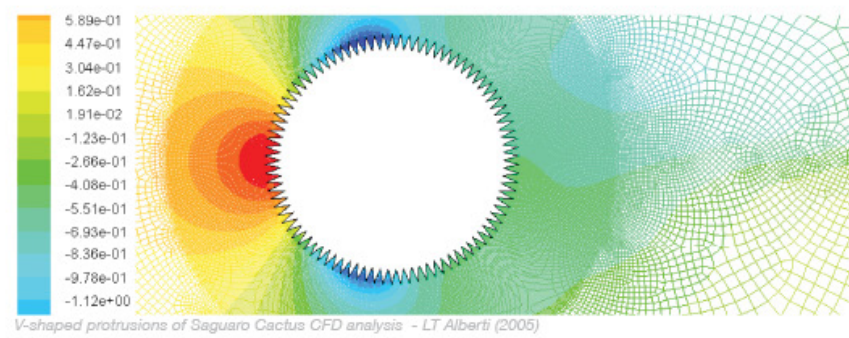

The sailfish skin consists of several $V$-shaped protrusic angled downstream inducing streamwise vortices.

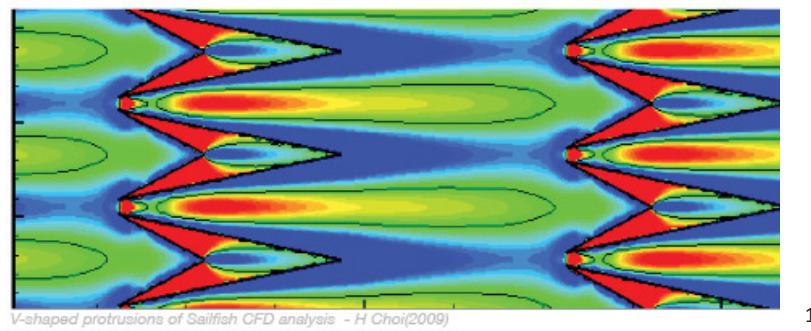

primarily focused on the macro building geometry with a focus on tall building performance primarily to study load shedding. To a lesser degree there were some secondary studies focused on interior airstream simulations in relation to human comfort. However, at the very end of the 20th century interest in how surface articulation affected building performance in the airstream began to emerge as an area of inquiry. Research published by Maruta, E., M. Kanda, and J. Sato $(1998)^{1}$ for example examined the effects of surface roughness on building performance at the end of last century. More recently, studies have been undertaken focusing specifically on how elements such as balconies affect building performance in terms of loading and energy performance as described by Lignarolo, Lorenzo, Charlotte Lelieveld, and Patrick Teuffel(2011)2 and H. Montazeri, and B. Blocken (2013) ${ }^{3}$.

In other fields of inquiry the relationship between surface roughness and issues of performance have been studied for some time. Perhaps most famously is how a shark's skin riblets produce counterintuitive friction reduction in water. Further exploration into how biological systems have developed surface articulation in similar ways uncovered two primary targets. Most notably the research of Haecheon Choi (2009) ${ }^{4}$ from the Center for Turbulence and Flow Control Research at the Bio-Mimetic Engineering Lab revealed two species that inspired further exploration in relation to buildings - the saguaro cactus and sailfish.

\section{PROBLEM}

The research presented here was instigated by questions raised at balcony scale across a building surface and then coupled with the bio-mimetic studies at a smaller relative scale to the overall object. This led to questions surrounding surface articulations in relation to building performance other than load shedding and to investigate more closely a finer scale of articulation at the building surface. Based initially on the research by Choi that suggests the saguaro cactus uses surface manipulations to reduce the effects of convective thermal transfer, the question became can a building do the same - reduce convective heat loss or gain simply through creating surface articulation? Of course, whereas manufacturing

Figure 1: Biological Case Studies for developing turbulence at surface. Left side the saguaro cactus and CFD of the cross-section turbulence. Right side a sailfish and CFD of surface turbulence. 
and aesthetic concerns for the cactus are evolutionary these become added dimensions of investigation for building cladding. A final constraint for the experiment would be the economics of the system. To achieve installation the outcome would need to be inexpensive materially while considering costs for labor and additional infrastructure; structure, fasteners, etc.

When taken together the material challenge would be to produce a lightweight yet dimensionally thickened surface while the aesthetic challenge would be to create continuity and variation across that same surface. The performance challenge is to, like the saguaro cactus, produce a surface that produces turbulence at the surface to create a layer of stagnant air that would reduce the exchange of heat energy to convection (e.g. Figure 1).

\section{DESIGN AND PRODUCTION}

The execution of this research was developed in two parallel tracks. One track being the study of the craft of the product; computational design to manufacturing, development of connections for installation, assembly details for water shedding, and pattern making for variation with minimal units. The next track being to study the ability of the surface to create turbulence along the surface developing the desired performance in a design that meets the aesthetic requirement. These two tracks would periodically unite to produce prototypes for testing.

Phase 1 - Cladding - Phase one began with a group of independent study exercises assigned to a small group of students to study producing a lightweight dimensional cladding system. Born out of project commission of the architecture firm E/Ye Design and brought into the academy as research, the emphasis for this first study was given to designing the physical details needed to develop a method for cladding. This initial prototype was limited to a 9" $\mathrm{x}$ 18 " dimension based on available manufacturing equipment found in the academic. Glass Fiber Reinforced Plastic was chosen as the initial material due to its strength to weight ratio and its ability to easily be cast into multiple shapes. Working with a composite manufacturing company, Windsor Fiberglass, these dimensional limitations were translated into numbers of molds that would be donated to facilitate production and testing. This partnership resulted in the production of a cladding system that had six unique unit types to generate desired performance and visual effects. The students consulted with the manufacturers to develop a tabbing system that would be built into the molded part and shingle to develop a positive drainage plane across the surface (e.g. Figure 2).

The geometric articulation for this cladding system drew inspiration from both the Saguaro Cactus and from the phenomena of drifting snow as this first prototype would be installed on a small building in a snowy climate. Low resolution CFD simulations done in Autodesk's Vasari were used to verify that the iterations of the geometry were indeed producing turbulence at the surface as shown in the lower left of figure 2.

After completing phase 1, it was concluded that the panel size of 9" x 18 " was too small to be economically viable as a deployable product. Additionally, this iteration did not account for corner detailing and therefor was not a complete cladding system. The lightweight panel and factory production did result in reduced installation time and labor costs as only 2 persons were needed for installation with no field cutting and zero on-site waste.

Phase 2 - Patterns and Edges -Based on the successes and failures of phase one the architecture firm and manufacturing company determined it was worthwhile to put more research into the product. Without having a commission for the another installation, phase two of the research was undertaken within a seminar course sponsored by the composite manufacturing company. Sponsorship, included support to produce four commercial grade molds and produce enough parts for a small installation within the School of Architecture 

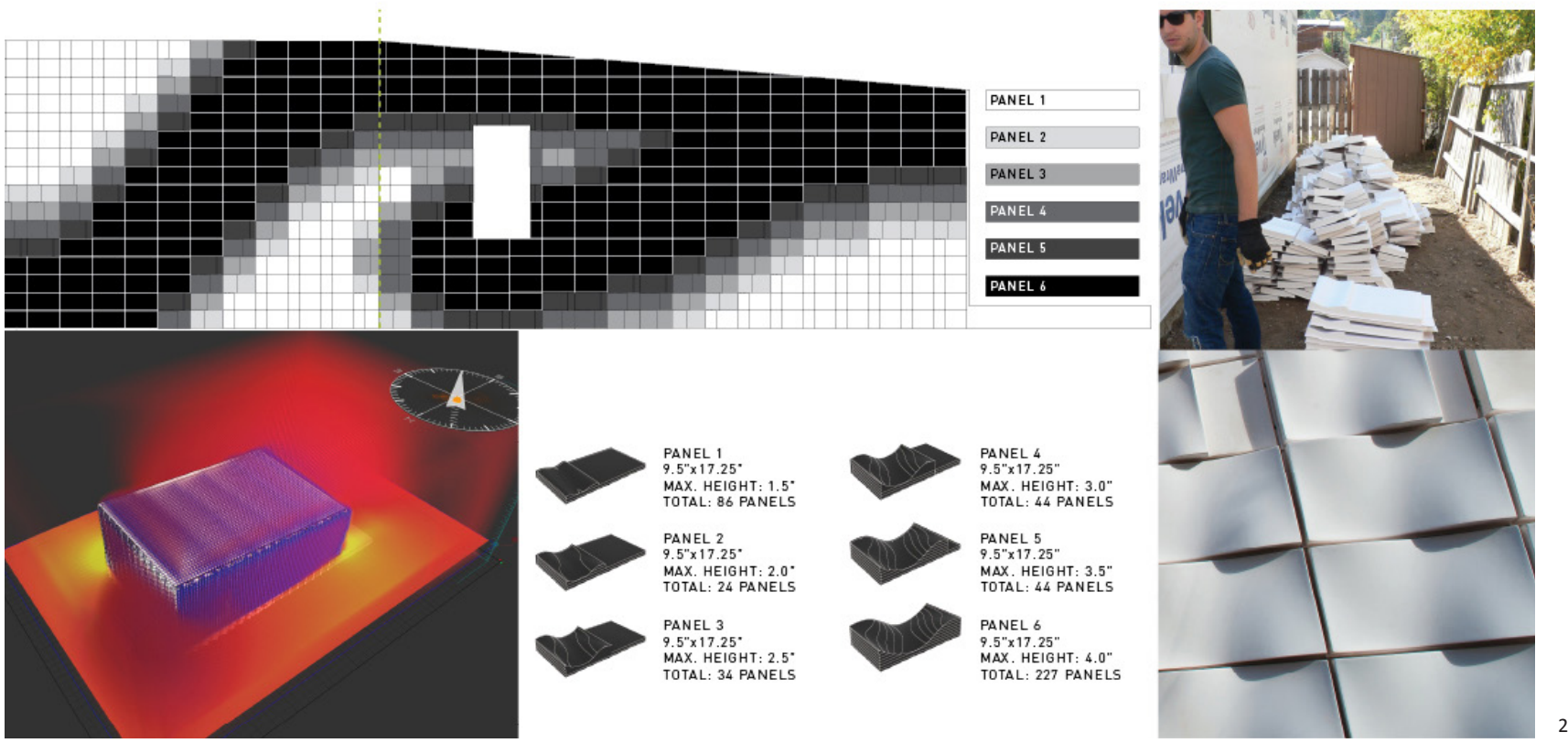

Salon. The focus here was to generate as much variation as possible and develop finished edges for the aggregated system with the allowed four molds. The students developed and tested several pattern studies until it was determined that a hexagon grid would be used. Two molds would be used for edge condition and two for the field. If the tabbing and connections were designed properly the two field units within the grid would each have six degrees of rotation giving the system an extreme amount of variation. Again working with the manufacturer the students produced the four patterns (positive plugs used to create molds) using the schools advanced CNC and manufacturing equipment designed with guidance in the form of constraints given by the manufacturer. Constraints such as draft angles for removing parts from molds, minimum radius of corners to ensure no cracking or fatiguing and level of finish for the final part would all be included by the students directly into their computational models that then were used to drive the CNC equipment to produce the patterns. The final installation was manufactured under the students direction, ordering proper numbers of all parts and providing labor for installation (e.g Figure 3 ).

Phase 3 - Corners and Scale - Phase three comes on the heals of phase 2 but this time with the support of another architecture firm, Liquid Design, commissioning the facade to be installed on a project of their design. Selected students from the previous phases were given internships to design a new system for the building facade installation. In collaboration with the architect and manufacturer it was determined that the larger the panel the more economic the product would be - with a limit being that it would be able to be carried by two crew members. However, as the mold increases in size the cost of manufacturing the mold increases dramatically. It was therefor determined that a single corner and single field part would be manufactured to reduce cost accosted with the mold production.

At this larger scale, a new design was needed. The inspiration for this project emerged from a return to the biological studies - in this case the sail fish (e.g. Figure 1) was chosen due to the more flowing graphic. The pattern generated on the surface of the sailfish was used as the inspiration to study a new articulation that would appear to move continuously along the length of the building while using only a single mold. While a sailfish is always oriented optimally to flow, a building will be confronted with any number of flow directions. Additionally, the corner research from phase 2 revealed that breaking up the corner vertically would relieve the generation of the large negative pressure build along the leading edge (e.g. Figure 4). 


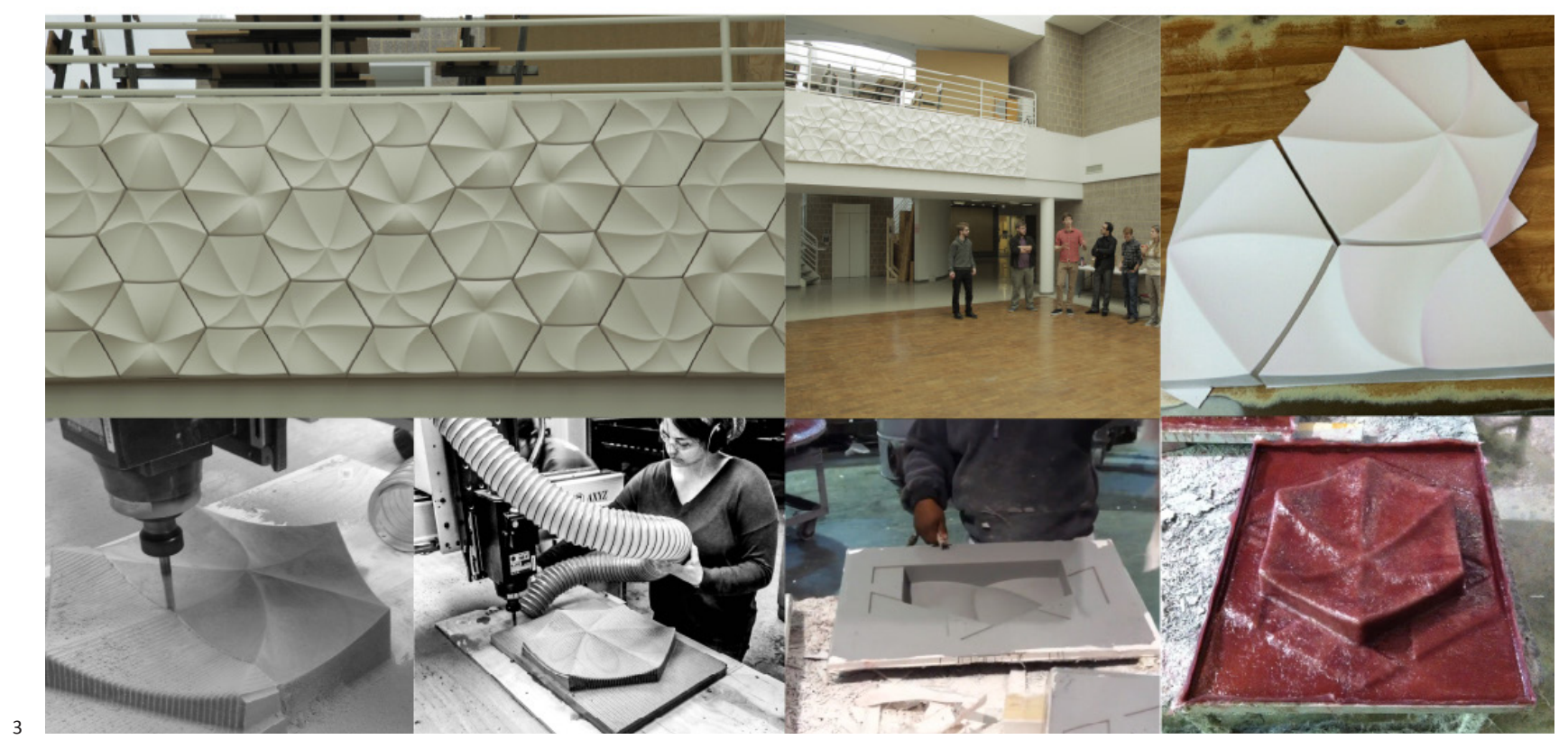

The results of phase three are a corner element 30 " tall $\times 54$ " along each edge and field panel $30 " x 144$. Due to its size a new material matrix had to be developed. The resulting material is a glass fiber reinforced plastic with a rovicore $@$ substrate to stiffen the part.

\section{MANUFACTURING}

In all three phases the students met with the manufacturers to work through the production process. Understanding the constraints of open mold hand layup for the first two phases provided great insight into issues of 'customized' mass production which according to the manufacturer are how they define part runs of less than 300 . In working with the manufacturer the academic responsibility was to produce the patterns that would be used to generate the molds for the finish parts. The pattern held all final requirements including finish as the parts would be used directly from the mold. Without post-processing other than trimming all issues associated with production had to be built in to the pattern such as ensuring the part would properly release from the mold while maintaining acceptable visual tolerances. Phase three introduced a new technique for production called Light Resin Transfer Molding (LRTM). This technique uses a closed molding system were vacuum channels are built into the mold to draw resin into the mold. This technique allows for greater control over part consistency and ultimately a stiffer part.

All phases within the study essentially entailed the same three major steps. The first step was handled wholly within the academic environment and included several rounds of consulting with manufacturers to produce the patterns for mold production. Patterns were milled on a 3-axis router from either MDF or high density foam. The team would then prime and seal the pattern with a hardened primer and hand finished to desired level of smoothness. The manufacturer would then take the pattern and produce the master mold by spraying the pattern with a hardened tooling gel coat followed by a very thick resin and glass layup. Finally, the manufacturer would begin running parts through these molds (either hand layup or LRTM) and trim the edges of the parts to specified requirements.

Figure 3: Phase 2 sponsored coursework. Top: final installation, presentation and molded parts from factory. Bottom: Milling patterns and mold for parts.installation.

It was here that the academy had the most impact on the manufactures assumed methods for production. Typically, the bulk of the financials are invested in the production of very expensive patterns to produce master molds when producing even moderately complex geometry. By using computer controlled manufacturing processes to generate the patterns instead of traditional methods, several thousands of dollars were saved and very precise 


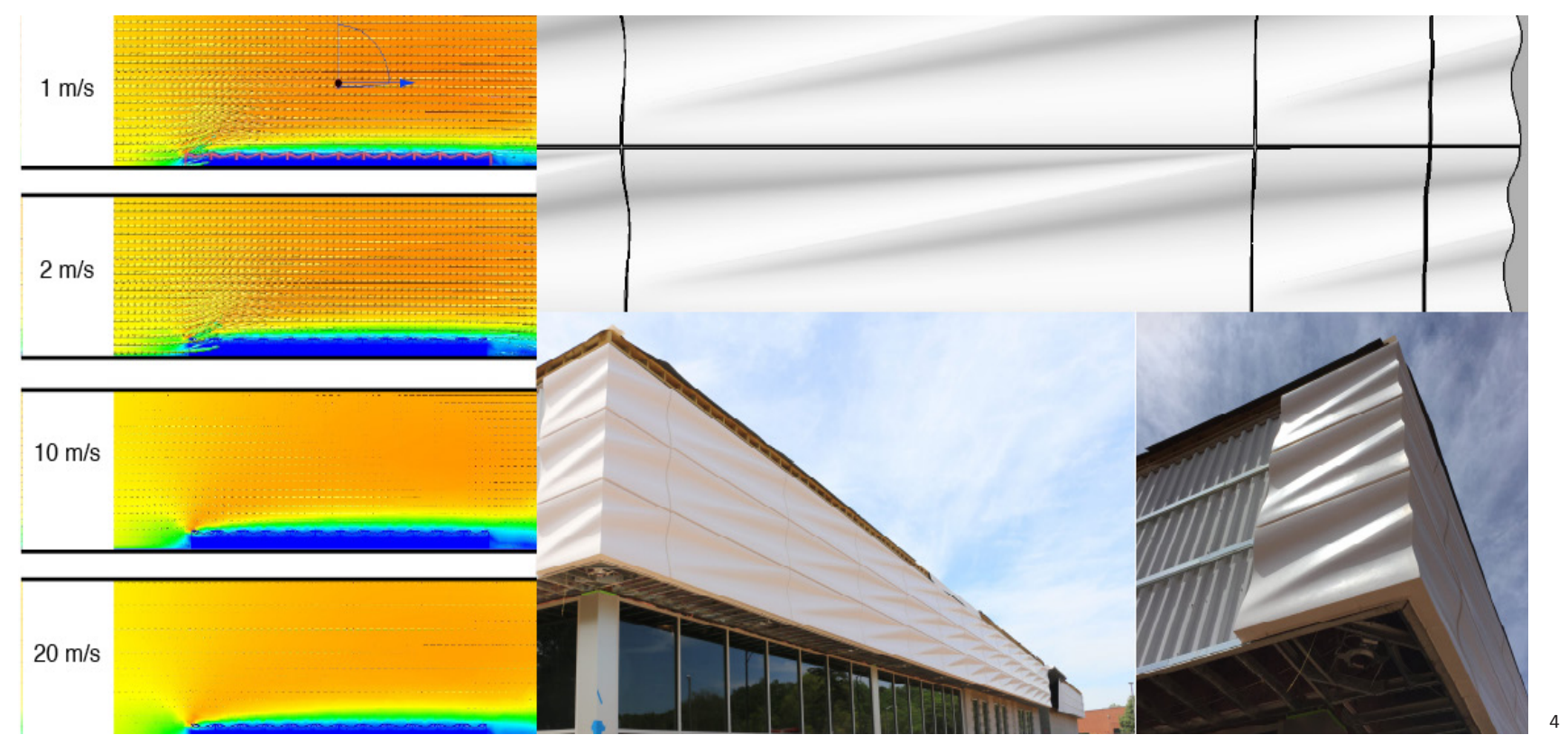

patterns were produced. There is some inherent risk as these patterns were destroyed in the mold making process but at a fraction of the cost of traditional pattern making the losses can easily be absorbed.

\section{DISCUSSION AND CONCLUSION}

Without a doubt the most successful part of this endeavor was working with a manufacturer to produce a finished, mass produced product that could be applied full scale to a building. In all three iterations the teams challenged some aspect of the manufacturing process to produce a more finished part than the previous iteration. This was only made possible by working closely with the manufacturer and architect to fully understand the constraints of their systems and how they could be translated into an architectural product. The success of this effort is demonstrated by the willingness of the Architects to engage the use of the product for their projects - convincing their clients that this technology and aesthetic would set them apart.

Turbulent Surfaces - While the initial computational fluid dynamic (CFD) simulations do indicate that a layer of turbulence is being generated by the geometric articulations verifying this in the field has proved difficult at full scale. This CFD testing of the cladding system confirms that the panels are environmentally activated through the interaction between the panel shape and the velocity of the wind -as airflows across the facade increase, the more turbulence the cladding geometry generates at the surface. This turbulence creates a thickening layer of 'stagnant' air along the building facade. The turbulent layer has an effect of adding an insulate airspace to buffer against the convective thermal transfer through the buildings facade. Additionally, this layer normalizes the pressure differences between interior and exterior when compared to a conventional flat facade. At lower air speeds the cladding allows the building to 'breath' as expected to avoid some issues associated with hermetically sealing a building. In this way the cladding acts more like a membrane allowing for a variety of exchanges, depending on climate and condition. Smaller scale models of the system will be needed to verify the development of turbulence at the skin in a wind tunnel. It is clear that if the turbulent layer is being generated at the surface of the building the heat exchange due to convection would be reduced - passively insulating the structure without hermetically sealing the entire building. 
Pattern Differentiation - There have been varying degrees of success with the aggregation of the pattern and generating variation with few unique parts. The first iteration relied on the most amount of unique parts but could be patterned to create an infinite amount of variation within the general aesthetic of that system. This version however, does not afford turning corners or elegantly terminating the run of parts. The second iteration of the research produced both incredible variation and allowed for the system to be terminated well. Parts were produced to turn corners as well though these were not incorporated into the final installation. However, performance as an exterior cladding system was compromised due to the modifications to the connectors that were developed to allow for the six degrees of rotation. The final iteration illustrated here in this paper does not afford nearly the variation in geometry of the previous studies but makes up for that in the two ways. One, the way the designed geometry produces a visualization that aggregates across the seam to blend the system into more of a whole - this gesture moves the eye in a different way than the gridded seams and maintains this visual play around the corner. Two, the subtle rolling of the geometry is interrupted with a crease to catch the sunlight in different ways creating visual variability through the day.

Cladding System - The efficiency of the system as a wall cladding system has continued to improve with each literation. Even at the largest scale, 36 " x 144", installation could be handled by a small crew of 3 without major heavy equipment. Phase 2 of the research proved to develop an incredible amount of variation with only 4 unit types but could not adequately shed water due to the necessary modifications made to the connections to allow for assembly. The final iteration proved a very efficient cladding unit with excellent environmental protection. Each unit covered 36 square feet of facade and could be installed at a very fast pace. The system shingles in the horizontal and laps in the vertical to positively drain water. Additional drainage channels are introduced within the vertical lap to prevent capillary creep behind the membrane.

At the moment, due to fire safety issues, the system can only be used for single story buildings less than $40 \mathrm{ft}$. However, with current research into resin compositions it will be possible to deploy lightweight systems made of Fiber Reinforced Plastics such as these on larger projects. Outside of the thermal benefits developed in this study through articulating the geometry, simply reducing the weight of a cladding system even a little will produce substantial savings by reducing building infrastructure to carry the facade. This not only reduces upfront costs but potentially embodied energy and total lifecycle cost for the building.

Future Considerations - In the end, working directly with a manufacturer for a specific installation was critical to the success of this research as imparting material expertise and production knowledge played a large part in the success of the project. Constraints driven by this relationship drove innovative solutions to achieve the aesthetic considerations for the project - namely producing visual differentiation without custom a price tag - while meeting performance goals. The partnership with the composites manufacturing company and the demands of practice have produced a secondary but important aspect of the system - the resultant solutions are extremely lightweight in comparison to typical claddings due to the material matrix. This means labor costs and installation time have been reduced dramatically which has afforded more capital investment into the design aspects of the system.

Patrick Teuffel. "Shape morphing wind-responsive
Parolo, Lorenzo, Charlotte Lelieveld, and facade systems realized with smart materials." Proceedings of the International Adaptive Architecture Conference, 2011.

3. Montazeri, H., and B. Blocken. "CFD simulation of wind-induced pressure coefficients on buildings with and without balconies: validation and sensitivity analysis." Building and Environment 60 (2013): 137-149.

4. Choi, Haencheon. "Bio-mimetic Flow Control." 62nd APS Division of Fluid Dynamics, 2009. 